\title{
Acute Myeloid Leukemia
}

\section{Abstract 2732}

Glasdegib in Addition to Intensive or NonIntensive Chemotherapy in Patients With Acute Myeloid Leukemia: Safety Analysis of Glasdegib 'On Target' Adverse Events

Jorge E. Cortes, Cristina Papayannidis, Catriona Jamieson, Gary J. Schiller, Anna Candoni, Brian Leber, Claudia D. Baldus, José A. Pérez-Simón, Weidong Wendy Ma, Corrado Gallo Stampino, Ashleigh O'Connell, Mirjana Zeremski, Geoffrey Chan, and Mikkael A. Sekeres

Visit http://www.bloodjournal.org/content/132/ Suppl_1/2732 for a complete list of contributor affiliations and full graphics.

Background: Novel agents are frequently added to standard acute myeloid leukemia (AML) treatment backbones yet it is unclear how much additional toxicity this introduces, with the potential for adverse events (AEs) to be caused by the backbone chemotherapy or the disease itself. Glasdegib (PF-04449913) is an investigational, oral small molecule inhibitor of the Hedgehog ( $\mathrm{Hh}$ ) pathway component Smoothened (SMO), currently in clinical development for AML treatment. In a Phase 2 randomized study, addition of glasdegib to lowdose cytarabine (LDAC) improved overall survival (OS) vs LDAC alone and combination therapy was generally well tolerated with minor differences in AE rates vs chemotherapy alone. Here, we analyzed specific 'on target' AEs consistent with inhibition of the SMO component of the Hh pathway to assess impact on overall toxicity.

Methods: We pooled safety data from both portions of a Phase $1 \mathrm{~b}+$ Phase 2 multicenter study (NCT01546038), including AML patients assigned to glasdegib $100 \mathrm{mg}$ QD with LDAC (non-intensive treatment) or with cytarabine/daunorubicin on a $7+3$ schedule (intensive treatment) (Figure 1). We assessed 'on target' all-causality treatment-emergent AEs of muscle spasms, alopecia, and dysgeusia.

J Adv Pract Oncol 2019;10(suppl 2):19-25

https://doi.org/10.6004/jadpro.2019.10.2.17
We also compared AE onset in defined study timeperiods (non-intensive: 0-6, 6-12, or >12 months; intensive: induction, consolidation, maintenance); defining long-term treatment as $>12$ months for non-intensive and maintenance for intensive.

Results: Across studies, 93 patients were enrolled in the non-intensive group and 80 in the intensive group. Here, we focused on patients treated with glasdegib: 89 patients in the non-intensive group and 78 in the intensive group. Table 1 shows baseline characteristics; median treatment duration was 69 days (range 3-1280) and 51 days (10-539), respectively. Rates of treatment discontinuation due to all AEs (inclusive of 'on target' and others), deemed by the investigator to be related to study drug (glasdegib and/or backbone chemotherapy) were similar (non-intensive, 10 patients [11.2\%]; intensive, 15 patients $[19.2 \%])$. Frequency of muscle spasms was similar for the 2 groups; observed in 19 of the nonintensive group (21.3\%) and 18 (23.1\%) of the intensive group (Table 1), with few grade 3 events (nonintensive $4.5 \%$; intensive $1.3 \%$ ), and the number of patients who developed muscle spasms (nearly all grade 1) when exposed to long-term treatment was small (4 non-intensive patients; 7 intensive patients) with no cases of rhabdomyolysis. Alopecia was reported for $8(9.0 \%)$ of the non-intensive group and $16(20.5 \%)$ of the intensive group (Table 1$)$. Alopecia had earlier time to onset in the intensive arm than in the non-intensive arm (Table 1), likely reflecting the concomitant chemotherapy. Alopecia was less frequent during glasdegib maintenance monotherapy (5.3\%) in the intensive arm than when given with $7+3$ (16.7\%). Dysgeusia was similar for the 2 groups, observed in 22 of the non-intensive group (24.7\%) and $24(30.8 \%)$ of the intensive group (Table 1). For both groups, dysgeusia had similar time to onset and was less common during long-term treatment. The number of patients having a dose modification as a consequence of class-related AEs was low (for nonintensive and intensive, respectively: dose reduction, $5.6 \%$ and $2.6 \%$; temporary discontinuation, $4.5 \%$ and $2.6 \%$; permanent discontinuation, $1.1 \%$ and $2.6 \%$ ). 


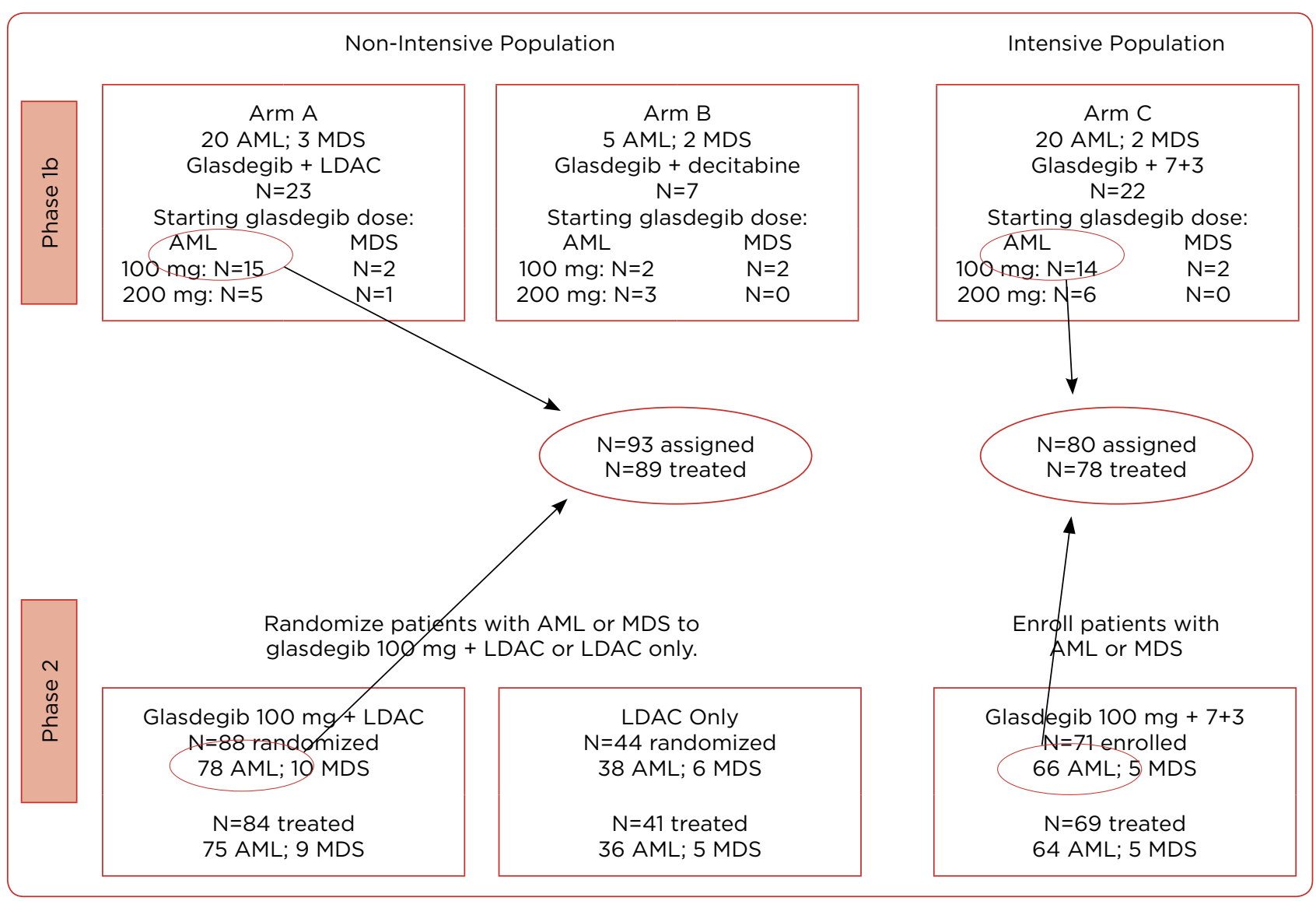

Figure 1. Study design: pooling of 'on target' patients. $A M L=$ acute myeloid leukemia.

Conclusions: Glasdegib 'on target' AEs of muscle spasms, alopecia, and dysgeusia were mainly of mild severity, had infrequent dose modifications or permanent discontinuations, and did not appear to impair tolerability of combination treatment. In comparison, muscle spasms and dysgeusia occurred in $\leq 5 \%$ for the LDAC alone arm, and no alopecia was reported. Although muscle spasms and dysgeusia occurred with similar frequency, dysgeusia occurred earlier, for a shorter duration, and was more persistent at the time of discontinu-

\section{The Advanced Practitioner Perspective: Lindsey Lyle, MS, PA-C}

The ASH annual meeting provides the opportunity for an endless amount of learning. With thousands of abstracts presented, it is impossible to attend every desired session. In the myeloid malignancy space, there is very exciting bench and clinical research that is making an impact on patient outcomes. As oncology ation compared with muscle spasms. Similar safety profiles were observed when combining glasdegib with LDAC or $7+3$, suggesting that glasdegib in combination with standard chemotherapy has a manageable safety profile and thus can be an acceptable combination partner in the treatment of AML. Our results are consistent with previously reported safety outcomes for glasdegib as monotherapy in hematologic malignancies. Clinical trials of glasdegib in combination with other standard of care AML agents are ongoing.

advanced practitioners (APs), it is critically important to be up to date on this research for the sake of our patients. Incorporating riskadapted treatment approaches, including sequential therapies, understanding novel agent mechanisms of action and associated side-effect profiles, and gaining knowledge on treatment durability and overall survival data for different treatment approaches is necessary to achieve optimal outcomes for our patients. 
In acute myeloid leukemia ( $A M L$ ), there have been several new and emerging therapies with a variety of mechanisms of action. This has significantly broadened the treatment landscape providing patients and health care professionals with more options and several targeted therapy approaches.

The three abstracts I discuss in my section focus on treatment strategies for patients with AML who are unfit for induction chemotherapy, identifying therapy-related on-target side effects, and understanding synergistic treatment approaches.

\section{Chemotherapy Plus Glasdegib}

Dr. Jorge Cortes presented Abstract 2732 on the safety and "on-target" adverse events with the addition of glasdegib to non-intensive or intensive chemotherapy in AML. In this phase $\mathrm{Ib}$ + phase II multicenter study, the investigators were looking at the addition of glasdegib in combination with either intensive chemotherapy or non-intensive chemotherapy. The intensive chemotherapy arm was cytarabine and daunorubicin in the standard $7+3$ administration, and the nonintensive chemotherapy was in combination with low-dose cytarabine.

One of the primary endpoints of this study was to identify potential on-target toxicity with the addition of glasdegib, a novel hedgehog inhibitor, to standard chemotherapy. As novel agents are being incorporated into treatment strategies in combination with a chemotherapy backbone, it is increasingly important to understand the additional side effects these new agents may cause. Namely, by understanding the mechanism of action, we can anticipate potential on-target toxicities. Glasdegib is an inhibitor of the hedgehog pathway. This pathway is responsible for tissue renewal and regeneration.

The known on-target side effects of glasdegib include alopecia, dysgeusia, and muscle cramps. Regarding these three mentioned side effects, there was no difference between the two arms except for alopecia, which was seen more commonly and more rapidly in the $7+3 \mathrm{arm}$; this was thought to be due to the backbone chemotherapy itself rather than the hedgehog inhibitor.

In regard to muscle spasms, the frequency was similar for the two groups, and mainly grade 1. There were few grade 3 events, with no cases of rhabdomyolysis. Rates of dysgeusia were also similar between the two arms, and mainly grade 1 . The time to onset of dysgeusia was similar, and it was less common during long-term treatment. The number of patients requiring a dose modification, treatment hold, or permanent discontinuation for on-target related AEs was low.

\section{Implications for the AP}

It is important for APs to familiarize themselves with the severity of toxicities seen in clinical trials, as with this combination therapy. Identifying the on-target side-effect profiles, educating our patients about the potential toxicities associated with these novel agents, and implementing supportive care strategies to help treat or mitigate the development of these adverse events will all help provide the best patient care.

Of great interest and excitement, based on results from the BRIGHT trial, glasdegib was recently FDA approved in combination with low-dose cytarabine. This combination was approved based on results reporting improved overall survival in combination with low-dose cytarabine alone vs. low-dose cytarabine alone in patients with $A M L$ in the upfront setting who are unfit for standard chemotherapy. We will be seeing an increasing number of patients taking glasdegib in our practices; educating and managing the ontarget side effects will be an important part of our role as advanced practitioners. 


\section{Abstract 285}

Venetoclax in Combination With Hypomethylating Agents Induces Rapid, Deep, and Durable Responses in Patients With AML Ineligible for Intensive Therapy

Daniel A. Pollyea, Keith W. Pratz, Brian A. Jonas, Anthony Letai, Vinod A. Pullarkat, Andrew Wei, Marina Y. Konopleva, Christian Recher, Olga Frankfurt, David Rizzieri, Tu Xu, Monique Dail, Brenda Chyla, Jalaja Potluri, and Courtney D. DiNardo

Visit http://www.bloodjournal.org/content/132/ Suppl_1/285 for a complete list of contributor affiliations and full graphics.

Background: Acute myeloid leukemia (AML) has a median age of diagnosis of 68 years; however, patients who are ineligible for intensive induction chemotherapy have limited therapeutic options. Venetoclax (Ven), an oral agent that targets the antiapoptotic protein, BCL-2, has shown synergistic antileukemic activity when combined with hypomethylating agents (HMA), decitabine (Dec) and azacitidine (Aza); this combination has resulted in high rates of durable remission, which are independent of cytogenetic and molecular characteristics.

Methods: This is an open-label, phase $1 \mathrm{~b}$, dose escalation and expansion trial (NCT02203773) studying the safety and efficacy of venetoclax in combination with decitabine or azacitidine. Patients had previously untreated AML and were ineligible for intensive chemotherapy due to comorbidities and age. Here, we present the data from the expansion cohort, where patients were treated with $400 \mathrm{mg}$ venetoclax in combination with either HMA. Venetoclax was administered daily in a three day ramp-up from 100 to 200 to $400 \mathrm{mg}$ and coadministered with either $20 \mathrm{mg} / \mathrm{m}^{2}$ of intravenous (IV) decitabine on days $1-5$ or $75 \mathrm{mg} / \mathrm{m}^{2}$ of IV or subcutaneous azacitidine on days 1-7 within each 28 day cycle. Dose adjustments for venetoclax were implemented for concomitant medications routinely used for prophylaxis with known drug-drug interactions. Safety and efficacy were evaluated. Time to first response, complete remission (CR), CR with incomplete blood count recovery (CRi), CR with partial hematologic recovery, duration of response, achievement of transfusion independence, overall survival (OS) and adverse events (AEs) were assessed. Minimal residual disease (MRD) was evalu- ated centrally by multicolor flow cytometry at a cutoff of $10^{-3}$ leukemic cells. Enrollment of patients treated with Ven + Aza began December 2014 and the majority was enrolled between January 2017 and June 2017; enrollment of patients treated with Ven + Dec occurred between November 2014 and June 2016, resulting in significantly different median follow-up time for the two treatment arms.

Results: Data cutoff was December 22, 2017. Of 115 patients treated with the $400 \mathrm{mg}$ dose of venetoclax, 84 were treated with Ven + Aza and 31 received Ven + Dec. The median ages for patients treated with Ven + Aza and Ven + Dec, respectively, were 75 (range: 61-90) and 72 (range: 65-86). Overall, 25\% and $29 \%$ had secondary AML, and $39 \%$ and $48 \%$ had poor cytogenetic risk, in patients treated with Ven + Aza and Ven + Dec, respectively. Transfusion dependence for red blood cells (RBC) or platelets within 8 weeks prior to venetoclax treatment was $64 \%$ (54/81) and 74\% (23/31) in patients treated with Ven + Aza and Ven + Dec, respectively. Key grade $\geq 3$ AEs across all patients were febrile neutropenia (44\%), anemia (28\%), pneumonia (25\%), thrombocytopenia (22\%) and neutropenia (18\%). Median time on study treatment was 6.4 and 5.7 months, and median follow up was 8.2 (range: $0.4-35.5$ ) and 16.2 (range: 0.7-36.7) months for patients treated with Ven + Aza and Ven + Dec, respectively. Key efficacy results are shown in the Table. Seventy percent and $74 \%$ of patients achieved CR/CRi, and the median time to first response was 1.2 and 1.9 months for patients treated with Ven + Aza and Ven + Dec, respectively. The median overall survival was 14.9 months for patients treated with Ven + Aza and 16.2 months for those treated with Ven + Dec. Among patients transfusion dependent at baseline, 52\% (40/77) achieved transfusion independence from both RBC and platelets, defined as not receiving $\mathrm{RBC}$ or platelet transfusion for $\geq 56$ days. Across both treatment groups, among patients with CR/CRi, $45 \%$ achieved MRD response less than $10^{-3}$ leukemic cells. Patients who received venetoclax dose reduction for CYP3A inhibitors had similar responses compared to those without dose reduction.

Conclusions: Venetoclax in combination with either azacitidine or decitabine led to high rates of rapid and deep responses that were durable in patients with AML ineligible for standard induction chemotherapy. A majority of patients who were 
transfusion dependent at baseline achieved transfusion independence after initiating venetoclax therapy. These results suggest that venetoclax, in com-

\section{The Advanced Practitioner Perspective: Lindsey Lyle, MS, PA-C}

Dr. Daniel A. Pollyea from the University of Colorado presented Abstract 285, on venetoclax in combination with hypomethylating agents in patients with AML who cannot receive intensive chemotherapy. The data for this abstract were based on results from a phase IB study, which was intended to evaluate the safety and efficacy of venetoclax (Ven) and hypomethylating agents (azacitidine [Aza] or decitabine [Dec]) in patients with acute myeloid leukemia who had not previously been treated, but unfit for standard chemotherapy due to either age or comorbidities. Venetoclax is an inhibitor of $\mathrm{BCL} 2$, which is an anti-apoptotic protein that is overexpressed in leukemia stem cells. There is known anti-leukemic synergy with venetoclax in combination with hypomethylating agents, which is why this strategy was implemented in patients with treatment-naive AML.

The results presented were on patients assigned venetoclax at a dose of $400 \mathrm{mg}$ po daily, after a 3-day ramp-up. Additionally, patients were either receiving azacitidine IV or SC for 7 days, or decitabine IV for 5 days with every cycle, which was every 28 days.

Dose adjustments of venetoclax were made for patients who were taking strong CYP3A4 inhibitors. Interestingly, within the context of this study, there was no identifiable decrease in the efficacy of the venetoclax or changes in overall outcomes with this dose reduction.

\section{Toxicities}

Key grade 3 toxicities seen with this combination therapy were febrile neutropenia, anemia, thrombocytopenia, neutropenia, and pneumonia. The toxicity profile was similar among both groups (Dec + Ven vs. Aza + Ven) and is similar to the most commonly experienced toxicities seen with standard induction chemotherapy for AML. bination with hypomethylating agents, may provide a potent therapeutic option for patients with AML who are not eligible for intensive chemotherapy.

The results reported from this study are impressive with regard to response rates and durability of response. The complete remission $(\mathrm{CR} / \mathrm{CRi})$ rates in patients treated with azacitidine and decitabine in combination with venetoclax were $70 \%$ and $74 \%$, respectively. Median time to first response was 1.2 months and 1.9 months, respectively. These results are remarkable, especially considering the fact that the median age of these patients was 73 years old.

\section{Durable Responses}

What this study also demonstrated was that these responses were durable. The current median duration of therapy was 14 months for the azacitidine arm and about 16.2 months for the decitabine arm. Due to the time at which the patients were enrolled, the duration of follow-up varied between the two arms. The median overall survival was 14.9 months for patients treated with Ven + Aza and 16.2 months for those treated with Ven + Dec. Among the patients who achieved a complete remission (CR/CRi), 45\% also achieved a minimal residual disease negative state, and as we know, this is an important factor for our patients' long-term outcomes.

Of clinical importance is the achievement of a transfusion-independent state, especially in patients who were transfusion independent prior to starting therapy. Among patients transfusion dependent at baseline, 52\% achieved transfusion independence from both red blood cells (RBCs) and platelets. This was defined as not receiving RBC or platelet transfusion for 56 days or longer.

The disease profiles of these patients in this abstract were comprised of a variety of molecular mutations and cytogenetic profiles. The presence of specific mutations/cytogenetic aberrations did not impact response to this combination therapy. 


\section{Abstract 2720}

Synergistic Anti-Leukemic Activity With Combination of FLT3 Inhibitor Quizartinib and MDM2 Inhibitor Milademetan in FLT3ITD Mutant/p53 Wild-Type Acute Myeloid Leukemia Models

Michael Andreeff, Weiguo Zhang, Prasanna Kumar, Oleg Zernovak, Naval G. Daver, Takeshi Isoyama, Kouichi Iwanaga, Noriko Togashi, and Takahiko Seki

Visit http://www.bloodjournal.org/content/132/ Suppl_1/2720 for a complete list of contributor affiliations and full graphics.

Background: MDM2, a negative regulator of the tumor suppressor p53, is overexpressed in several cancers including hematological malignancies. Disrupting the MDM2-p53 interaction represents an attractive approach to treat cancers expressing wild-type functional p53. Anticancer activity of small molecule MDM2 inhibitor milademetan (DS-3032b) has been demonstrated in preclinical studies and in a phase 1 trial in patients with acute myeloid leukemia (AML) or myelodysplastic syndrome. Quizartinib is a highly selective and potent FLT3 inhibitor that has demonstrated single-agent activity and improvement in overall survival in a phase 3 clinical study in relapsed/ refractory AML with FLT3-internal tandem duplication (FLT3-ITD) mutations. We present here the preclinical studies exploring the rationale and molecular basis for the combination of quizartinib and milademetan for the treatment of FLT3-ITD mutant/TP53 wild-type AML.

Methods: We investigated the effect of quizartinib and milademetan combination on cell viability and apoptosis in established AML cell lines, including MV-4-11, MOLM-13 and MOLM14, which harbor FLT3-ITD mutations and wild type TP53. Cells were treated with quizartinib and milademetan at specified concentrations; cell viability and caspase activation were determined by chemiluminescent assays, and annexin $\mathrm{V}$ positive fractions were determined by flow cytometry. We further investigated the effect of the combination of quizartinib and the murine specific MDM2 inhibitor DS-5272 in murine leukemia cell lines $\mathrm{Ba} /$ F3-FLT3-ITD, Ba/F3-FLT3-ITD+F691L and Ba/ F3-FLT3-ITD+D835Y, which harbor FLT3-ITD, ITD plus F691L and ITD plus D835Y mutations, respectively. F691L or D835Y mutations are associated with resistance to FLT3-targeted AML therapy. In vivo efficacy of combination treatment was investigated in subcutaneous and intravenous xenograft models generated in male NOD/SCID mice inoculated with MOLM-13 and MV-4-11 human AML cells.

Results: Combination treatment with milademetan (or DS-5272) and quizartinib demonstrated synergistic anti-leukemic activity compared to the respective single-agent treatments in FLT3 mutated and TP53 wild type cells. Combination indices (CIs) were $0.25 \pm 0.06,0.61 \pm$ $0.03,0.62 \pm 0.06,0.29 \pm 0.004$ and $0.50 \pm 0.03$, respectively, in MV-4-11, MOLM-13, MOLM-14, Ba/ F3-FLT3-ITD+F691L and D835Y cell lines, all of which harbor FLT3-ITD or ITD plus TKD point mutations. The combination regimen triggered synergistic pro-apoptotic effect in a p53-dependent manner as shown by annexin- $\mathrm{V}$ staining and caspase $3 / 7$ assays. Mechanistically, the combination treatment resulted in significant suppression of phospho-FLT3, phospho-ERK and phospho-AKT and anti-apoptotic Bcl 2 family proteins (eg, Mcl-1), as well as up-regulation of p53, p21 and pro-apoptotic protein PUMA, compared to single agent treatments. Of note, the combination regimen also exerted a synergistic pro-apoptotic effect on venetoclax (BCL-2 inhibitor)-resistant MOLM-13 cells (CI: $0.39 \pm 0.04$ ) through profound suppression of Mcl-1. In an in vivo study using the MOLM-13 subcutaneous mouse xenograft model, quizartinib at 0.5 and $1 \mathrm{mg} / \mathrm{kg}$ and milademetan at 25 and $50 \mathrm{mg} / \mathrm{kg}$ demonstrated a significant tumor growth inhibition compared with vehicle treatment or respective single-agent treatments. In MV-4-11 intravenous mouse xenograft model, the combination of quizartinib plus milademetan showed a significantly prolonged survival, with no animal death in the combination group during the study period, compared to respective single agent treatments and untreated control (Figure).

Conclusion: Synergistic anti-leukemic activity was observed for quizartinib plus milademetan combination treatment in preclinical AML models. A phase I clinical trial of quizartinib/milademetan combination therapy in patients with FLT3-ITD mutant AML is underway. 
The Advanced Practitioner Perspective: Lindsey Lyle, MS, PA-C

Dr. Michael Andreeff from MD Anderson Cancer Center presented Abstract 2720, on the synergistic anti-leukemic activity combining the FLT3 inhibitor quizartinib and the MDM2 inhibitor milademetan in FLT3+/TP53 WT AML models.

\section{Quizartinib Plus Milademetan}

MDM2 is overexpressed in AML and other hematologic malignancies, and it is a negative regulator of TP53. The investigators studied the synergistic activity of these two agents (the FLT3 inhibitor quizartinib and the MDM2 inhibitor milademetan) in murine models with established AML cell lines expressing the FLT3ITD mutation and wild-type TP53. Quizartinib has single-agent activity and has been shown to improve overall survival in patients with FLT3-positive disease with an ITD mutation. Quizartinib is currently being further investigated in phase III trials. The MDM2 inhibitor milademetan has been used in preclinical studies and in a phase I clinical trial in patients with AML and MDS. Milademetan is notable for having an anticancer activity.

With both agents having single-agent activity, the goal of the researchers, as stated in this abstract, is to determine if combining these agents promotes a synergistic and more potent apoptotic effect on leukemic stem cells. Phase I clinical trials with quizartinib and milademetan are currently ongoing in patients with FLT3-ITD mutant, TP53 wild-type AML.

\section{Key Points for APs}

The most important theme for advanced practice providers at this stage of clinical development is to start thinking about sequential therapy in patients with AML. In the age of multiple novel agents with molecular/pathway targets, APs should familiarize themselves with options for second- and third-line therapies, for example, identifying the next potential treatment option if a patient with FLT3+ AML is perhaps resistant to the FLT3 inhibitors that are currently available. Perhaps this synergistic approach, which was shown to target FLT3 mutations known to be resistant to traditional FLT3 inhibitors in these mouse models, may come to fruition in our patients as well.

Interestingly, in preclinical studies, this combination therapy has shown a synergistic proapoptotic effect on stem cells that are resistant to venetoclax (BCL-2 inhibitor), which may be of significant clinical importance. Again, it is critical for APs to understand the current research landscape, gain knowledge regarding the use of this and other combination therapies in the clinical setting, and identify appropriate sequential therapies. We will certainly have more to come, especially as novel agents continue to be incorporated into clinical trials and specifically, as this phase I trial accrues and progresses into further clinical development. 